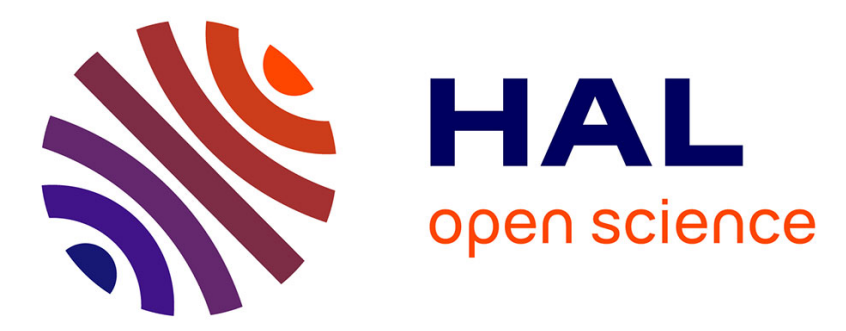

\title{
Survey of correlation properties of polyatomic molecules vibrational energy levels using FT analysis
}

\author{
Rémi Jost, Maurice Lombardi
}

\section{To cite this version:}

Rémi Jost, Maurice Lombardi. Survey of correlation properties of polyatomic molecules vibrational energy levels using FT analysis. 2nd International Conference on Quantum Chaos and 4th International Colloquium on Statistical Nuclear Physics, 1986, Cuernavaca, Mexico. pp.72, 10.1007/3-540-171711_5. hal-01023551

\section{HAL Id: hal-01023551 \\ https://hal.science/hal-01023551}

Submitted on 14 Jul 2014

HAL is a multi-disciplinary open access archive for the deposit and dissemination of scientific research documents, whether they are published or not. The documents may come from teaching and research institutions in France or abroad, or from public or private research centers.
L'archive ouverte pluridisciplinaire HAL, est destinée au dépôt et à la diffusion de documents scientifiques de niveau recherche, publiés ou non, émanant des établissements d'enseignement et de recherche français ou étrangers, des laboratoires publics ou privés. 


\title{
SURVEY OF CORRELATION PROPERTIES OF POLYATOMIC MOLECULES
}

VIBRATIONAL ENERGY LEVELS USING F T. ANALYSIS

\author{
R. JOST and M. LOMBARDI \\ Service National des Champs Intenses (C.N.R.S.) \\ BP $166 \mathrm{X}, 38042$ Grenoble Cédex, FRANCE. \\ and \\ Laboratoire de Spectrométrie Physique (U.S.T.M.G.) \\ BP 87,38402 Saint Martin d'Hères Cédex, FRANCE.
}

\section{Introduction}

In the last few years molecular spectroscopists have begun to study the highly excited vibrational levels of polyatomic molecules. In this high energy regime vibrational quantum numbers can no longer be intrinsically assigned (in contrast with vibrational levels at low energy). One can only characterize these levels by their correlation properties(1), we shall consider:

- Short range correlations which are characterized by the Next Neighbor Distribution, (N.N.D.). These correlations range from a POISSON (random or uncorrelated spectra) to a WIGNER distribution (which shows "level repulsion").

- Long range correlations are characterized by the $\sum 2(L)$ and $\Delta_{3}(L)$ function. They describe the behavior which ranges from an uncorrelated spectra (POISSON statistic) to a spectra with "spectral rigidity".

In nuclear physics, spectra obtained many years ago by low ener gy neutron scattering show the phenomena of "level repulsion" and "spectral rigidity" (1). These results have stimulated theoretical developments such as Random Matrix Theory (R.M.T.) (2) and the Gaussian Orthogonal Ensemble (G.O.E.) (2). In the field of molecular physics the theoretical predictions have preceeded experimental results. Many classical trajectory calculations of two-dimensional systems show features of "irregular" behaviour(3). These classical trajectories have also been calculated with the potential energy surfaces of the electronic ground state of triatomic molecules. All these calculations suggest a smooth change of the dynamics from regular to irregular behavior as the energy exceeds a certain threshold. At an intermediate energy regime, the phase space is embedded with both regular and irregular regions. Quantum calculations with models 
of (usually) two degrees of freedom, produce eigenvalues which display strong correlations properties(4). Very few quantum calculations have employed the realistic potential surfaces of polyatomics, due to the prohibitive size of the matrix to be diagonalized. Even calculations concerning the simplest polyatomic, triatomic molecules involve three vibrational degrees of freedom. Comparison of the classical and the quantum calculations for two dimensional systems, show a qualitative agreement, i.e., the correlation properties of eigenvalues evolve from POISSON to G.O.E. in the same energy range where the classical trajectories display a transition from "regular" to "irregular" behavior(5). Up to now, there is no quantitative relationship between these classical and quantum descriptions.

The main interest in molecular physics for these problems arise from the need to understand the phenomenon of vibrational energy redistribution, which is the basis of the usual R.R.K.M. Theory for unimolecular reactions. This theory supposes complete intramolecular thermalisation of vibrational energy before reaction (the isolated molecule acts as its own "thermal bath"). The basis of this theory is believed to be that at relevant excitation energy all classical trajectories are ergodic. A few classical trajectories addressing directly this problem for two-dimensional systems have been made recentiy $(6)$.

In this paper we will summarize in chapter $V$ the experimental results obtain to date concerning the statistical correlation properties of the vibrational energy levels of polyatomics. First we present, in chapter II, a general review of the properties of vibrational energy levels and, in chapter III, their relationship with expe rimental molecular spectra. In chapter IV the Fourier Transform method is presented as a tool to displays correlations in experimental spectra.

II. General considerations on the vibrations of polyatomic molecules.

In this section, we shall only consider molecules without rotation (see III). The number of vibrational degrees of freedom, i.e. the number of vibrational modes for non linear species is $N=3 n-6$ (for $n \geqslant 3$ ), where $n$ is the number of atoms (diatomics have only one vibrational mode and they are not considered here). For each electronic state, the bottom of the $N$ dimensional potential energy surface can be approximated by $N$ harmonic oscillators. This means that at "low" energy, the vibrational levels are well characterized by the 
normal modes description, except for the occurence of an occasional Fermi resonance. When we consider levels at high energies, $E$, the density of states increases as $\varphi(E) \propto[E /\langle\omega\rangle]^{N-1}$ where〈w> is the geometric mean of the frequencies. Typically, the magnitude of $\langle\omega\rangle$ is of the order of $1000 \mathrm{~cm}^{-1}(0.12 \mathrm{eV})$ and the height of the well ranges from $10^{4} \mathrm{~cm}^{-1}$ to $\mathrm{few} 10^{4} \mathrm{~cm}^{-1}$ (a few ev) for the ground state of stable molecules. Consequently, there is a very rapid increase in the vibrational density of states with energy and with the number of atoms. The coupling terms in the Hamiltonian, like higher order cross terms in the potential or kinetic energy operators induce an increasing number of Fermi resonance as the density of states becomes larger. The absence of a complete set of spectroscopic constants, or accurate ab initio potential energy surfaces, precludes any precise calculation of eigenvalues at high vibrational energies. Nevertheless a model of oscillators coupled with cubic and quartic cross terms in the potential, with a harmonic basis set can be considered. This model yields a very sparse matrix. The validity of such a model stems from the spectroscopy of the lower excited vibrational levels which shows a very rapid decrease of matrix elements with increasing the order of the cross terms. The size of the cubic coupling matrix elements may be as large as a few hundred $\mathrm{cm}^{-1}$ in the particular case of stretch-bend $\mathrm{C}-\mathrm{H}$ coupling but, most of the matrix elements are much smaller: 0.1 to $10 \mathrm{~cm}^{-1}$ for cubic and quartic coupling terms. We expect from rough calculations on random matrix (7) that the mechanism of overlapped Fermi resonances plays a crucial role in terms of statistical properties when the density of states becomes iarger than 1 to 100 levels per $\mathrm{cm}^{-1}$, depending on the molecule. The effect of these coupling on vibrational energy redistribution has been studiedby techniques which depend mainly on the phenomenon of mixing of wavefunctions (observed via probability of transitions), not on energy level positions. The various vibrational modes which are mixed at a given energy emit different spectra, which may be used to label them (at least qualitatively)(8). Some more recent work use picosecond time resolved spectroscopy to study these couplings(9). The conclusion of such recent studies is that one can find lack of vibrational redistribution at surprisingly high energy in large molecules, increasing the interest to find other measures of these coupling on relevant molecules and at relevant energies. On one hand, the fast convergence of the polynomial expansion in harmonic coordinates may be no longer valid for large amplitude vibrational motion. A contrario, 
for a large molecule, a great amount of vibrational energy, enough to dissociate it for example, can be attained by distributing the energy over the large number of modes available with only very few $(0,1,2)$ quanta in each mode. This kind of vibrational level, named "combination level", which correspond to very harmonic motion at large energy, is in fact the vast majority of states present at energies interesting for chemical studies like photochemistry, intermolecular vibrational relaxation, and multiphoton excitation. But the minority (in terms of numbers) of very anharmonic states which concentrate the energy on one given mode (for example a local mode which leads to dissociation) play probably an important role in chemical process like dissociation, isomerization, fluorescence decay, etc...

\section{Experimental technique on molecular spectra.}

According to usual ideas coming from previous work of nuclear physicists, in order to study the statistical correlations between levels, it is necessary to consider only those levels which have the same good quantum numbers (spin, parity and total angular momentum). Thus the spectroscopic method must be able to sort these levels by their quantum numbers in order to avoid the superposition of sets (see chapter IV). In addition, the spectrum must contain nearly all the levels in a given energy range (no "missing" levels). The experimental signal to noise ratio must be large enough to let all the levels appear with sufficient intensity. The spectrum should yield a complete set of levels, therefore the resolution should be sufficient to avoid overlapping lines (no blended lines). Only gas phase laser spectroscopy is able to produce molecular data at high vibrational excitation with a typical resolution of $\mathrm{MHz}$ (for c.w. laser) to $\mathrm{GHz}$ (for pulsed laser) required for statistical analysis.

Nevertheless, most of experimental molecular spectra do not satisfy the requirement cited above. It is why, a new technique of analysis, the Fourier Transform described in chapter IV has been evolved to deal with experimental spectra which suffers imperfection.

This new technique, which takes full advantage of the fact that large (but imperfect) stretches of levels can be obtain, clearly opens new avenues to analyze the large amount of data which can be obtained in molecular physics in chemically interesting situations.

The classical spectroscopic techniques relevant to study the vibrations of polyatomic molecules are summarized as follows: 


\section{A. Infrared spectroscopy (vibrational excitation).}

This technique has produced a great deal of information about the fundamental vibrational frequencies and the overtones of $\mathrm{CH}$ and $\mathrm{OH}$ stretches in the ground state. However infrared studies at high vibrational excitation are restricted to mainly these types of overtones and then do not allow to observe the numerous "combination" levels since the transitions occur between levels on the same potential surface. Furthermore, rotational congestion occurs and precludes to observed dense sets of levels (see B, below).

\section{B. Excitation spectroscopy (electronic excitation).}

In this technique, transitions from the vibrationless electronic ground state $\left(S_{0}\right)$ to the (first) excited state $\left(S_{1}\right)$ allow to study the rovibronic levels in the excited state. But Franck Condom factors do not permit the observation of every vibrational level because many of them are too small.

Furthermore, as in I.R. spectra, rotational congestion occurs: at room temperature, there is a large number of rotational levels populated in the molecule as the rotational constants range from $0.1 \mathrm{~cm}^{-1}$ to a few $\mathrm{cm}^{-1}\left(10^{-4}-10^{-3} \mathrm{eV}\right)$. Even though the selection rules $(\Delta \mathrm{J}=0, \pm 1) 1 \mathrm{imit}$ the number of rotational transitions, the number of rotational lines appearing in the spectra is pretty large (typically $10^{2}$ to $10^{3}$ per band). These lines are spread over a region of about $100 \mathrm{~cm}^{-1}\left(\approx 10^{-2} \mathrm{eV}\right)$. This spread is much larger than the mean spacing of vibrational levels we want to study (see above) and consequently precludes the observation of every vibrational level. The use of a supersonic jet (a free expansion of a mixture of a carrier gas (He,Ar...) with the molecule of interest) cools the rotational temperature to about $1 \mathrm{~K}$ for a few $M \mathrm{sec}$. The corresponding rotational spectrum is much less congested as few rotational levels are populated in the vibrationless ground electronic state. But, even with a supersonic jet, the possibility of the overlap of rovibronic bands remains (see $\mathrm{NO}_{2}$ results below).

A further spectral selectivity is possible with an additional spectroscopic step: double resonance technique, such as Stimulated Emission Pumping (S.E.P.) or Microwave-Optical Double Resonance (MODR) and also Anticrossing Spectroscopy (A.S.) are possible techniques for producing spectra corresponding to a simple set of good quantum numbers. For example, in S.E.P., a transition from the vibrationless 
ground state populate a single rovibronic level $(\mathrm{J}=0$ for example), in the excited state. Then a second transition, by stimulated emission, transfer population, according to well defined selection rules, down to one (or very few) rotational levels of high vibrational levels of the ground state. The spectral simplicity achieved by this method allows to consider each line (or set of very few lines) of the spectrum as a vibrational eigenvalue with a known angular momentum. We shall present three examples of results mainly obtained by double resonance method in $\mathrm{NO}_{2}$, Acetylene $\left(\mathrm{C}_{2} \mathrm{H}_{2}\right)$ and Methylglyoxal $\left(\mathrm{CH}_{3}-\mathrm{O}-\mathrm{C}-\mathrm{C}-\mathrm{H}-\mathrm{O}\right)$.

A very related probiem is the coupling between rotational and vibrational degrees of freedom. Two examples concerning $\mathrm{NO}_{2}$ and $\mathrm{H}_{2} \mathrm{CO}$ will be discussed at the end of the $\mathrm{N}_{2}$ chapter. Beforehand, in chapter IV, we present a new method of analysis of the correlation properties of spectra : the Fourier transform.

IV. The Fourier Transform: a new method to analyse the correlation properties of spectra.

The statistical method like N.N.D. and $\Delta_{3}$ have been deveropped in order to analyse the available data in nuclear physics, i.e. a relatively small set of levels (typically 50 per nucleus). These levels constitute a high quality data set with respect to signal to noise, resolution and spectral purity (single $\mathrm{J}^{\pi}$ assignment). Then, these data have been analysed with the N.N.D. and $\Delta_{3}$ in terms of stick spectrum. In a recent paper, L. Leviandier et al (10) introduce the Fourier Transform (F.T.) as a tool to measure statistical correlation properties able to treat a noisy, poorly resolved and spectrally impure spectrum. In this method, the raw spectrum is Fourier transformed, without extracting a stick spectrum, to obtain a function $c(t)$. The correlation properties can be determined from the smoothed, ensemble averaged, square of the modulus of $c(t), i . e \cdot \overline{c(t)} 2$. Consider a spectrum composed of lines with the line shape $L(f)$. The amplitude of lines is assumed to be the product of a deterministic envelope $A_{E}$ by a a stochastic function $A S$ of the position. Then, $\overline{\mid c(t)} 2$ contains two components as sketched in fig. 1 .

a) The "fast component", which is the square of the F.T. of the envelope $A_{E}$, is proportional to $N^{2}$, i.e. the square of the nomber of levels. This "fast component" gives a very large peak at the origin. For example, it is a $(\sin N t / t)^{2}$ function when $A_{E}$ is a rectangle function. 

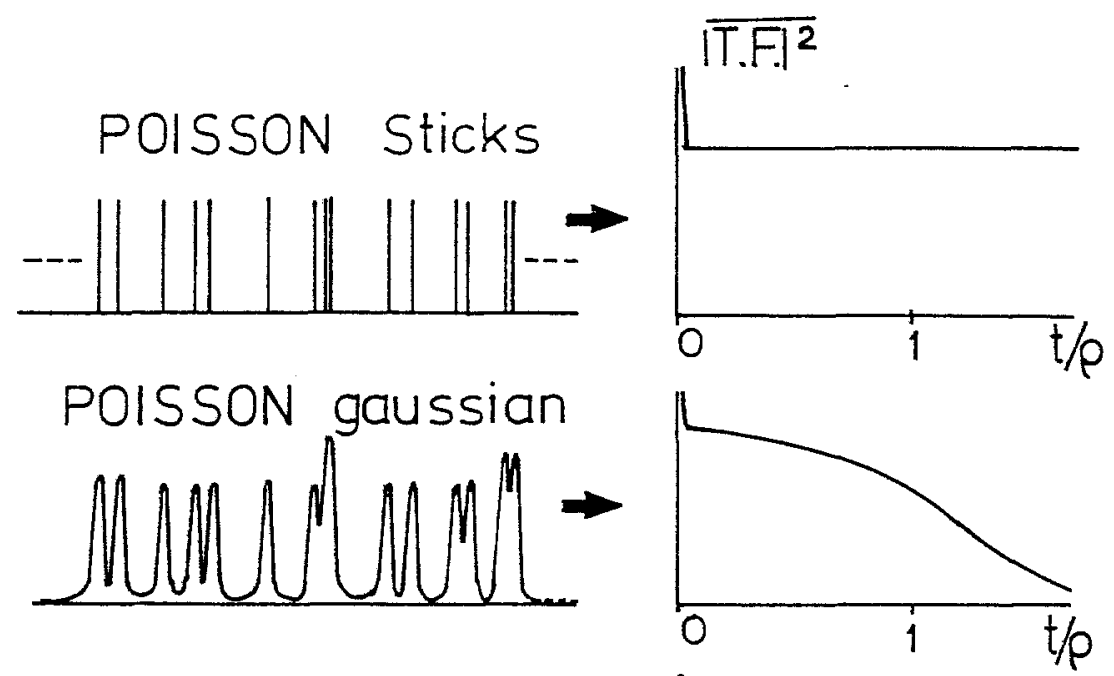

G.O.E. Sticks
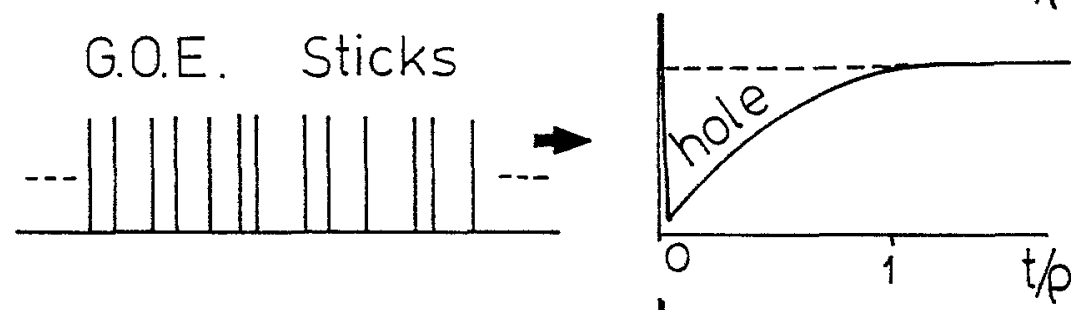

G.O.E. gaussian lines

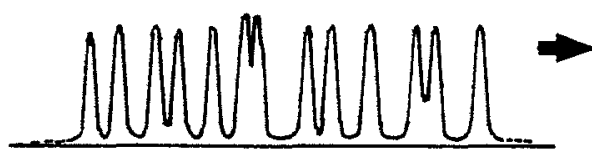

finite length correlations Sticks
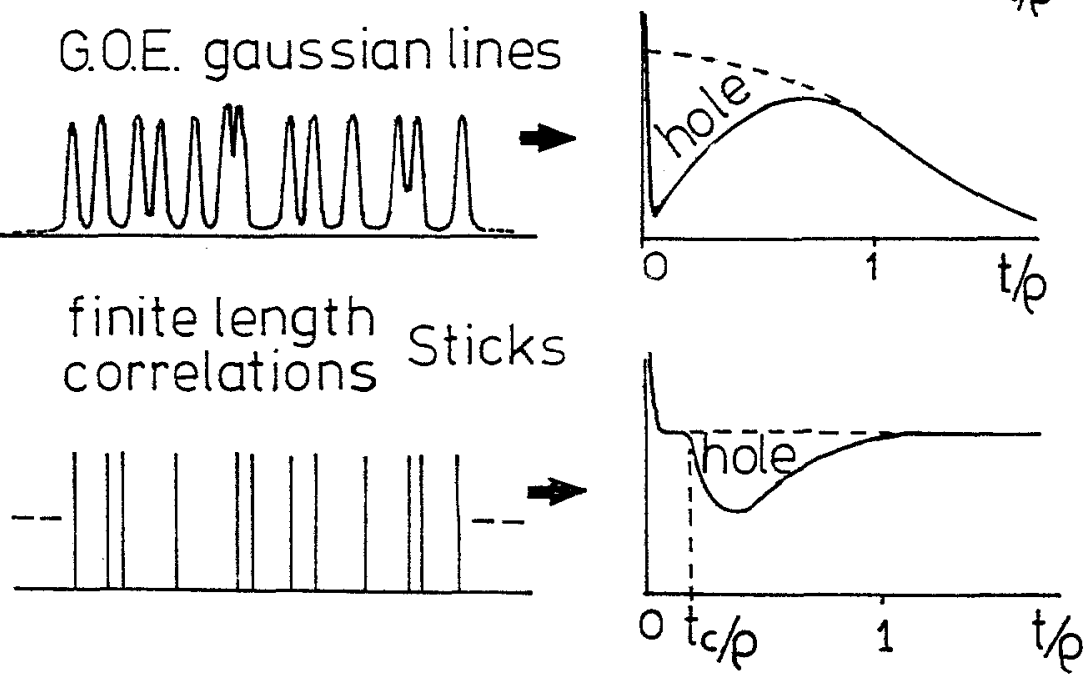

Fig. 1 : Typical spectra and their corresponding smoothed (or ensemble averaged) square of Fourier Transform |F.T|2. The "fast component" appears near the origin. Unsmoothed |F.T 2 displays $100 \%$ fluctions as shown in figure 4 (down). the is a dimensionless variable, where $\rho$ is the density
of lines. 
b) The "slow" component is composed of the F.T. of the individual lineshapes $L(f)$. Its amplitude is proportional to $\mathrm{N}$.

Furthermore, if there are correlations in the spectrum, the shape of $\overline{|c(t)|} 2$ changes after the fast component : a "correlation hole" appears. This can be explained in the following way: the random position of lines, $f_{j}$, are described by one and two level correlation functions: $R_{1}(f)=d$ which is the level density, and $R_{2}\left(f_{1}, f_{2}\right)$ which is the joint probability that there is a level at $f_{1}$ and another level at $f_{2}$. The two levels correlations can be expressed as $R_{2}\left(f_{1}, f_{2}\right)=R_{1}\left(f_{1}\right) \cdot R\left(f_{2}\right) \cdot\left(1-Y_{2}\left(d \cdot f_{1}, d . f_{2}\right)\right)$ where $Y_{2}$ is the two levels cluster function given by Metha(2). If $Y_{2}$ is not zero, i.e. if correlations exist, the "slow component" is multiplied by $\left[1-b_{2}(t)\right]$, where $b_{2}(t)$ is the F.T. of $Y_{2}$. The shape of $\left[1-b_{2}(t)\right]$ is shown on figure 1 for two limiting cases: POISSON $\left(b_{2}(t)=0\right)$ and G.0.E. The decrease of the amplitude of $1-b_{2}(t)$ near the origin (for $t / p<1$ ) is called a "correlation hole". The shape of this "hole" depends on the particular system and experimental situation. Furthermore, for any random spectrum (Poisson or G.O.E), the F.T. of a single spectrum is $100 \%$ randomily modulated with a frequency of the order of the reciprocal of the width of the envelope. This random modulation is mathematically analogous to the speckle phenomenon familiar to laser users and it can be reduced either by an ensemble averaging or by smoothing of the spectrum or both.

The relationship between spectra and its $\overline{|c(t)|} 2$ is shown for typical examples in figure 1 .

Some comments are necessary in order to better understand the meaning of the F.T. of a spectrum and espacially why correlations properties can been seen even when the spectrum is "bad" (see above).

First this F.T. method, looks familiar for molecular physicists dealing wint the theory of radiationless transitions in "intermediate" molecules. Consider phenomena like intramolecular transfer of energy between a single vibrational non stationary state which can be directly excited by a pulse of light (with a laser) and a dense manifold of "dark" (i.e. for which there are no optical transitions) vibrational states. This dense set may belongs to the same electronic state (pure vibrational energy transfer) or an other electronic states with the same electronic spin (internal conversion to the ground 
state) or with different spin multiplicity (intersystem crossing between singlet and triplet). The "doorway state" which carry oscillator strength is "diluted" by coupling between a lot of neighbouring states, giving a spectrum composed of a lot of lines whose amplitude are the product of a deterministic envelope $A_{E}$ which represente the smoothed energy dependence of the amount of the doorway state contained in each the molecular eigenstate, and a stochastic component because the coupling can be considered as random. The time evolution of the physical system is then gouverned by two components. A sufficiently short burst of light (a picosecond laser pulse) excite the pure (non stationary) doorway state which is in fact a coherent superposition of a stretch of stationary molecular eigenstates. All these eigenstates start to radiate in phase, but, since they have differents frequencies, they dephase in a time of the order of the reciprocal of the width of $A_{E}$, giving the "fast component". After this time the molecular eigenstates radiate incoherently giving the slow component whose time constant is the reciprocal of the width of individual spectral line (1ifetime). The interesting point is the relationship between the correlations properties of eigenstates and theirs corresponding time evolution. This question has been addressed by Delory and Tric as early as in 1974(11) but up to now, no example of correlation hole has ever been observed in time resolved experiments. Only biexponential decay corresponding to a POISSON statistics have been reported. The speckle "noise" on the slow component, which can also be called many levels quantum beats, seems to have been overlooked in time resolved experiments because the fluorescence decay is smoothed, due to poor time resolution and/or superposition of several decay corresponding to simultaneous observation of different rotational levels.

As second comment, it is intersting to notice the relationship between the correlation hole of the F.T. and the $\Sigma 2(L)$ function used as standard test of long range correlations.

The shape of the correlation hole can be numerically related with the shape of the $\Sigma^{2}(L)$ function. For example, the ordinate and the slope at the origin of the slow component is related with the POISSON and G.O.E. contribution of the $\Sigma 2(L)$ function for large $L$. We conclude that the correlation hole of the F.T. of a spectrum is mainly a measure of long range statistical correlations properties of this spectrum. This explains why the F.T. is relatively insensitive 
to lack of resolution in the spectrum : long range correlations properties are lost, in principle, only if resolution is worse than the considered range of these properties. By contrast N.N.D. informations are rapidly lost as soon as the resolution is worse than the average level spacing.

As third comment, the superposition of $m$ independent G.O.E. spectra produces a correlation hole m times narrower. The same informations about correlations can be recovered if one F.T. a stretch containing m times more levels. This explain why this F.T. method allows to display correlations even in impure spectrum which can be considered as the superposition of independent G.0.E.'s. This point is to be contrasted with autocorrelation or N.N.D. techniques for which the correlations properties, like level repulsion, disappears with only two independent G.O.E.

At this point we should remark that the superposition of independent set of levels may be due to profound and interesting physical reasons like for example when there is a strong anharmonic coupling inside subsets of modes of a molecule but not coupling between these subsets (this mean that there are remaining good quantum numbers).

As a result the $F . T$. method enables to measure the number of independent subsets, by measuring the ratio of the time corresponding to the onset of the correlation hole to the time corresponding with the level density (mean spacing).

As fourth comment, for a given "pure" subset, "intermediate coupling", i.e., coupling much smaller than for G.0.E. leads to finite range of the correlations properties. This gives a very characteristic shape to F.T. (see fig. 1). The correlation hole exist only between $t=\varphi$ and $t_{c}, t_{c}<t_{0}$, where $t_{c}$ is a characteristic time for vibrational redistribution.

We emphasize the difference with G.0.E. for which $t_{c}$ is basically 0 . This $t_{c}$ can be measured even for "bad" spectra as soon as the stretch of levels is larger than a few times reciprocal of $t_{c}$.

The crucial point was to found an integral method to measure long range properties, avoiding the step of defining individual components (the extraction of a stick spectrum from the experimental spectrum) which cannot be done when the resolution is worse or comparable with the average spacing of lines.

As a conclusion, the "correlation hole" is the signature of the correlation properties. 
Examples of F.T. of experimental spectrum are given below.

\section{Experimental results.}

\section{A. Excitation Spectrum and Microwave Double Resonance of $\mathrm{NO}_{2}$}

In 1975 Smalley et al (12) have obtained the excitation spectrum of $\mathrm{NO}_{2}$, rotationally cooled $\left(T_{R}=3 \mathrm{~K}\right)$ in a supersonic jet in the region of $6708 \mathrm{~A}-5708 \mathrm{~A}$, i.e. from $14900 \mathrm{~cm}^{-1}$ up to $17500 \mathrm{~cm}^{-1}$ above the vibrationless electronic ground state. They observed 140 vibrational bands. The strong non adiabatic interaction between $2 \mathrm{~A}_{1}$ and ${ }^{2} B_{2}$ explain the main features of the observed spectrum(13). We discuss here the statistical analysis done by Haller et al (14) on this data set for which they found a wigner statistics for N.N.D. First, in the range of 14900-16580 $\mathrm{cm}^{-1}$, there are 20 "hot bands" identified among the 83 bands observed ("hot bands" originate from excited vibrational level(s) of the electronic ground state). In the remaining region of the spectrum (16580-17500 $\left.\mathrm{cm}^{-1}\right)$ there are probably numerous hot bands; thus, there is a total of the order of 35 "hot bands" among the 140 observed bands. Second, each band consists of 10 to 50 rotational $1 i-$ nes which spread over $10 \mathrm{~cm}^{-1}$. The mean spacing between the bands is $19 \mathrm{~cm}^{-1}$, thus many bands overlap and give a high probability that two bands are considered as one. This effect would produce a spurious "level repulsion". For example, in reference (12), there are many more 1 ines in bands number 113 and 22 than in other bands (see bands number 115 and 95 for instance). This is a strong indication that bands number 113 and 22 are composed of at least two overlapped vibrational bands. As a conclusion the validity of the statistical analysis done by Haller et al on $\mathrm{NO}_{2}$ is doubtful because there are too many spurious and missing levels. Furthermore, this analysis demonstrates the necessity to obtain a pure data set and consequently to use double resonance techniques.

Recently, Lehmann and Coy(15) have obtained, by a microwaveoptical double resonance technique, a very dense spectrum of $\mathrm{NO}_{2}$. An optical transition to high excited vibrational level at $16800 \mathrm{~cm}^{-1}$ to $17100 \mathrm{~cm}^{-1}$ above the vibrationless ground state level was excited. Specific rotational levels $(J=7$ to 10$)$ are selected with a microwave rotational transition in the vibrationless $2 \mathrm{~A}_{1}$ ground state. The number of observed transitions is a factor of 8 greater than expected if only the allowed rotational transition to any $B_{2}$ symmetry vibronic 1 evel were observed. Hardwick(16) has suggested that the numerous 
Transitions from $10(0,10)$ level of $\mathrm{NO} 2$

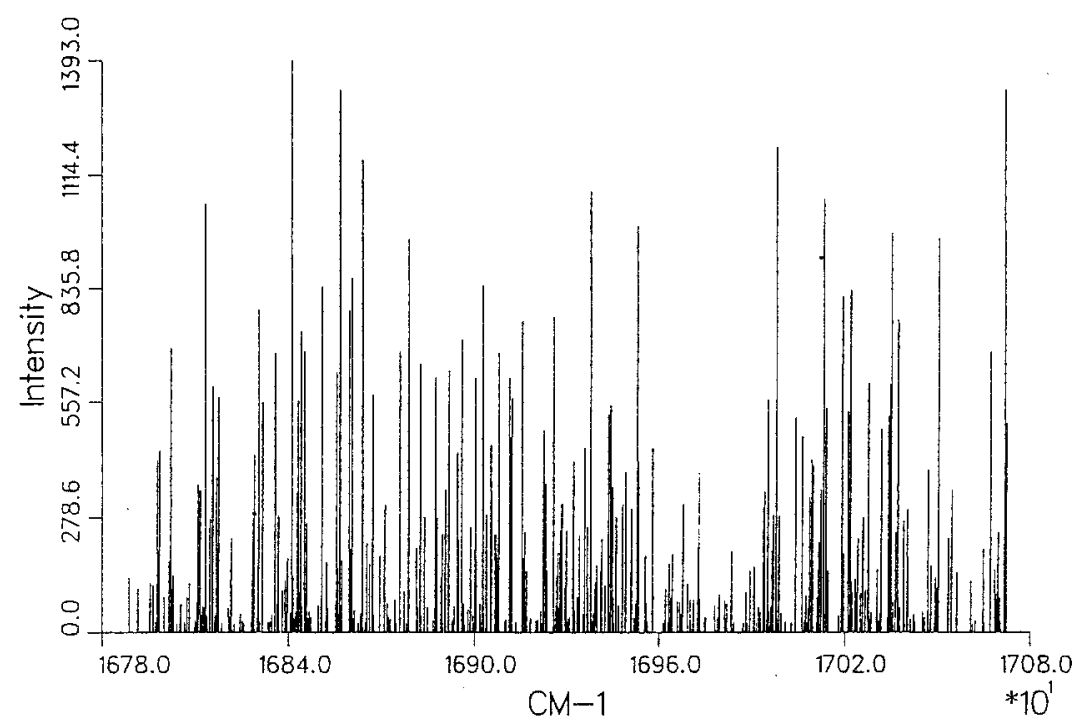

Fourier Transform of the $10(0,10)$ level of NO2

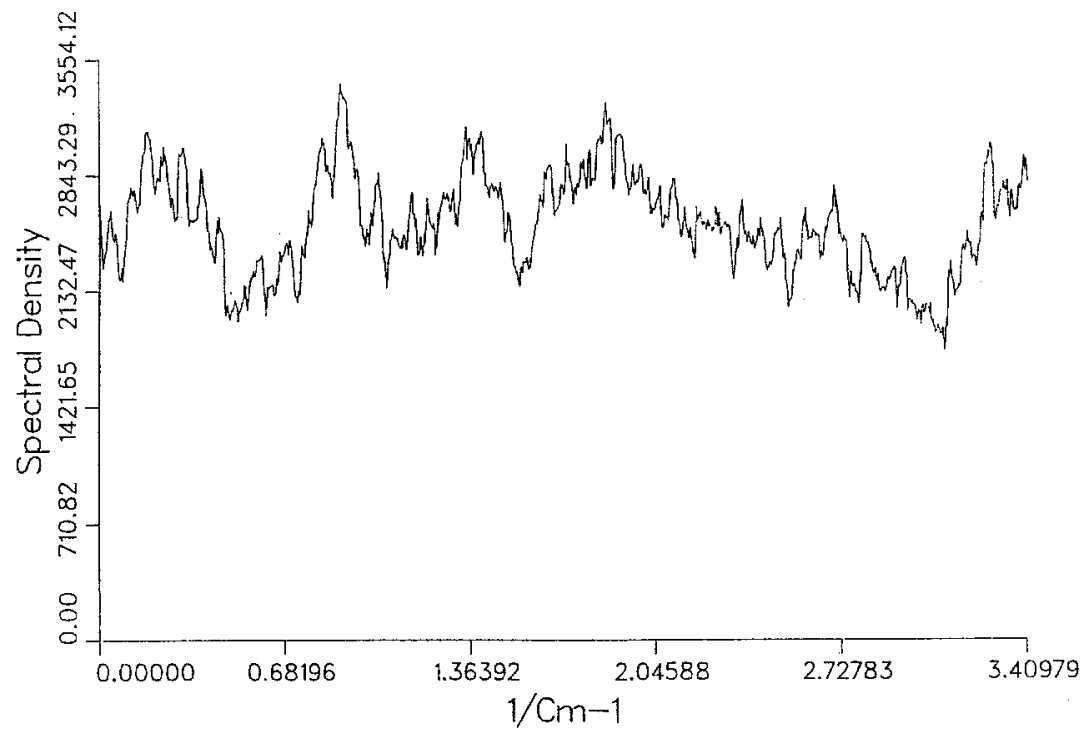

ig. 2 : Stick experimental spectrum of M.O.D.R. of $\mathrm{NO}_{2}$ and the corresponding, partly smoothed $|F . T| 2$. There is no correlation hole near the origin. 
forbidden transitions observed are related to quantum ergodicity in these highly excited vibrational levels. The number of observed lines is a factor of 3 less than the number predicted if all selection rules wer broken as predicted by Hardwick. However, the analysis of these spectra by the F.T. method, as described above, displays no correlations (17) as shown of $f i g .2$. This breaking of rotational selection rules may also be explained by a molecular axis switching instead of vibrational coupling.

\section{B. S.E.P. spectra on formal dehyde $\mathrm{H}_{2} \mathrm{CO}$}

Very similar results have been obtained by the S.E.P. technique at MIT by H.L. DAI et al (18) on $\mathrm{H}_{2} \mathrm{CO}$ : the only lines which appeared in the spectra at 1 ow values of the rotational quantum number $(J \leqslant 3)$ are those expected. At $h i g h e r ~ J$ and $K_{a}$ rotational quantum numbers, the spectra rapidly become more complex and the observed level densities at $\mathrm{J}=10, \mathrm{~K}=2$ are several times larger than the known total density of vibrational levels. This increase in the density of accessible vibrational levels was the result of a rotation induced mixing of the anharmonic vibrational basis function (Coriolis coupling) which compromised the "goodness" of both vibrational and $\mathrm{K}_{\mathrm{a}}$ quantum numbers. A further analysis of these results, with a criterion for chaos developped by Heller et al (19) shows that the increasing complexity in the spectrum with increasing angular momentum corresponds nonetheless with decreasingly chaotic behavior. This can be understood in terms of an available phase space volume that expands more rapidly than the occupied phase space volume as $J$ increases.

In conclusion one can study the "pure" vibrational coupling by considering $\mathrm{J}=0$ levels and study rotational-vibrational coupling (Coriolis) by studying higher $J$ (and $K$ ) rotational levels.

\section{Stimulated Emission Pumping (S.E.P.) spectra of acetylene.}

In 1983, Abramson et al (20) have observed, by S.E.P., very high vibrational energy levels (at $27900 \mathrm{~cm}^{-1}$ ) of the ground electronic state of acetylene. A pulsed dye laser (the PUMP) excites (at $45300 \mathrm{~cm}^{-1}$ ) a specific rovibronic level of the $1_{\mathrm{A}}$ excited electronic state. A second pulsed dye laser (the PUMP) stimulates transitions 
down to high vibrational levels of the $1 \times \mathrm{xg}$ ground state. A decrease of the total fluorescence of the upper level is observed when the frequency of the PUMP 7 aser is in resonance with a transition to a $1 \times g$ high vibration level. They observe a new type of vibrational behavior. The transitions are organized in clump of typically $501 i-$ nes with the same $J$ values. The analysis of the spacings between adjacent 1 ines (N.N.D.) from a stick representation of the spectrum shows that they approximatively follow a Wigner distribution in each clump. Further analysis by Mukamel et al (21) shows that there are missing levels in the stick spectrum, either due to the limited resolution which forbids the observation of too close levels or because the weakest lines are hidden in the noise. The analysis of the intensity distribution according to a modified Porter-Thomas distribution gives an estimation that $70 \%$ of the levels are missing ! On the other hand, analysis with the $\Delta_{3}$ statistics indicates that only about $15 \%$ of the levels are missing. Further analysis by Sundberg et al (22) gives $75 \%$ and $6 \%$ of missing levels for intensity distribution analysis and $\Delta_{3}$ statistics respectively. Clearly more experiments, especially at higher resolution, are required in order to avoid missing levels. But the Fourier Transform (see chapter IV) of the original spectrum as displayed in figure 3 shows that a strong correlation exists in the original spectrum. The strong correlation found in the stick representation of the acetylene spectrum, which might be due to a subjective analysis, is confirmed by the Fourier Transform technique.
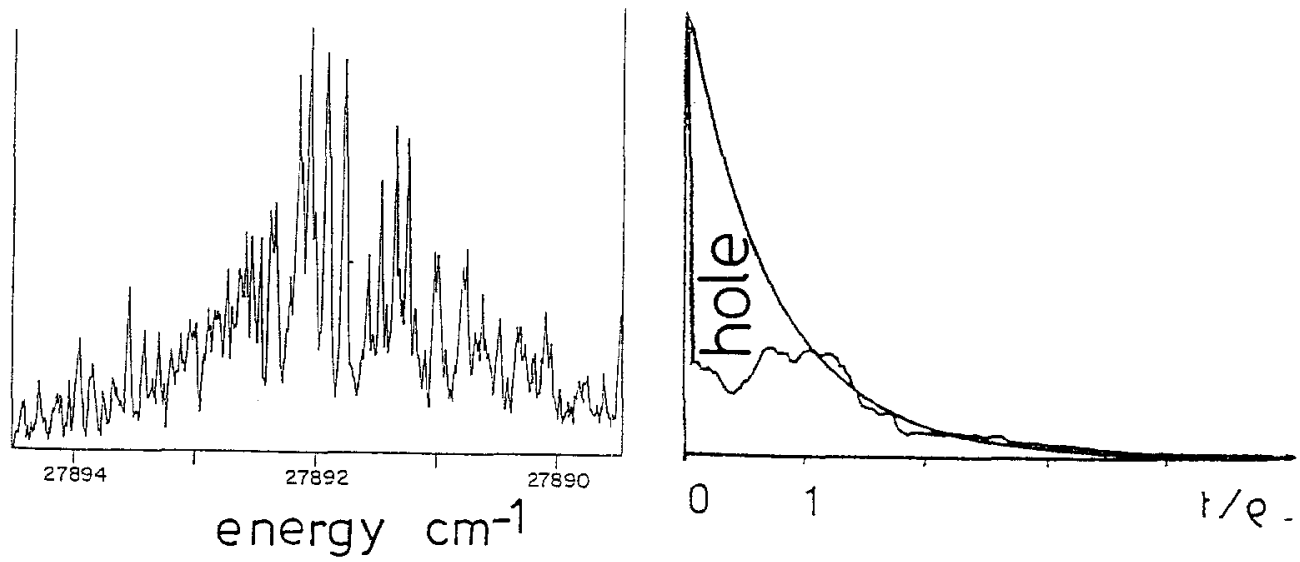

Fig. 3 : S.E.P. Acetylene spectrum near $27900 \mathrm{~cm}^{-1}$ and the corresponding smoothed, Fourier Transform ( $\mid$ F.T $\mid 2)$ which displays a correlation hole. A hole appears also on the $|F . T| 2$ of the corresponding stick spectrum. 
D. Anticrossing spectra of glyoxal $\mathrm{C}_{2} \mathrm{H}_{2} \mathrm{O}_{2}$ and methylglyoxal.

In the singlet-triplet anticrossing technique, a (strong) magnetic field is applied in order to Zeeman tune triplet levels into resonance with a single rovibrational level of a singlet electronic state. The anticrossing is a lorentzian, which is detected as a decrease in the fluorescence of the excited singlet. A strong magnetic field, provided by the Service National des Champs Intenses (S.N.C.I.) (Grenoble, France) allows one to obtain many anticrossings (and vibrational levels). Our Bitter coil gives a field up to 8 Tesla, which allows the observation of numerous triplet vibrational levels within $\pm 7 \mathrm{~cm}^{-1}$ of the selectively laser excited singlet level of $S_{1}$. The ground electronic state $\left(S_{0}\right)$ of glyoxal (or methylglyoxal) is a singlet, and the first triplet state, $T_{1}$, is at lower energy than the first singlet excited state $S_{1}$. At the energy of the vibrationless level of $S_{1}$, the corresponding vibrational density of states of triplets, $T_{1}$, is $\approx 1$ level per $\mathrm{cm}^{-1}$ for glyoxal, and $\approx 201$ evels per $\mathrm{cm}^{-1}$ for methylglyoxal (see fig. 4). A supersonic jet of glyoxal is locates within the $100 \mathrm{~mm}$ bore diameter of the Bitter coil. A single rotational $(N=0)$ vibrational level of $S_{1}$ is selectively excited by c.w. ring dye laser. In contrast with an optical or microwave spectrum, all of the anticrossings have the same amplitude (a decrease of up to 50 \% of the fluorescence intensity). The only "missing" anticrossings are due to magnetic field inhomogeneity broadening (1 Gauss: $0.7 \mathrm{MHz}$ ), natural linewidth (23), and overlapping anticrossings.

Consequently, we expect only a few missing levels in the anticrossing spectrum.

For glyoxal, the width of the anticrossings varies over three orders of magnitude, from $\approx 1$ gauss to $\approx 10^{3}$ gauss. These widths are related to the magnitude of the singlet-triplet spin-orbit vibronic coupling. The excitation of the rotationless $N=0$ singlet levels avoid the superposition of several anticrossing spectra which correspond to different Zeeman sublevels. According to the first order singlet-triplet anticrossing selection rules due to spin-orbit vibronic coupling $(24)$, only triplet $(S=1)$ rotational level with $N=1$, $M_{N}= \pm 1, M_{S}=\mp 1$ (and $K=0$ or $K=1$ according to the vibrational symmetries) can anticross the $N=0$ excited singlet. The distribution of the widths (as contrasted with the distribution of line intensities in the spectra considered previously) results in a more complex shape for the "slow component" of the F.T. of the spectrum. 

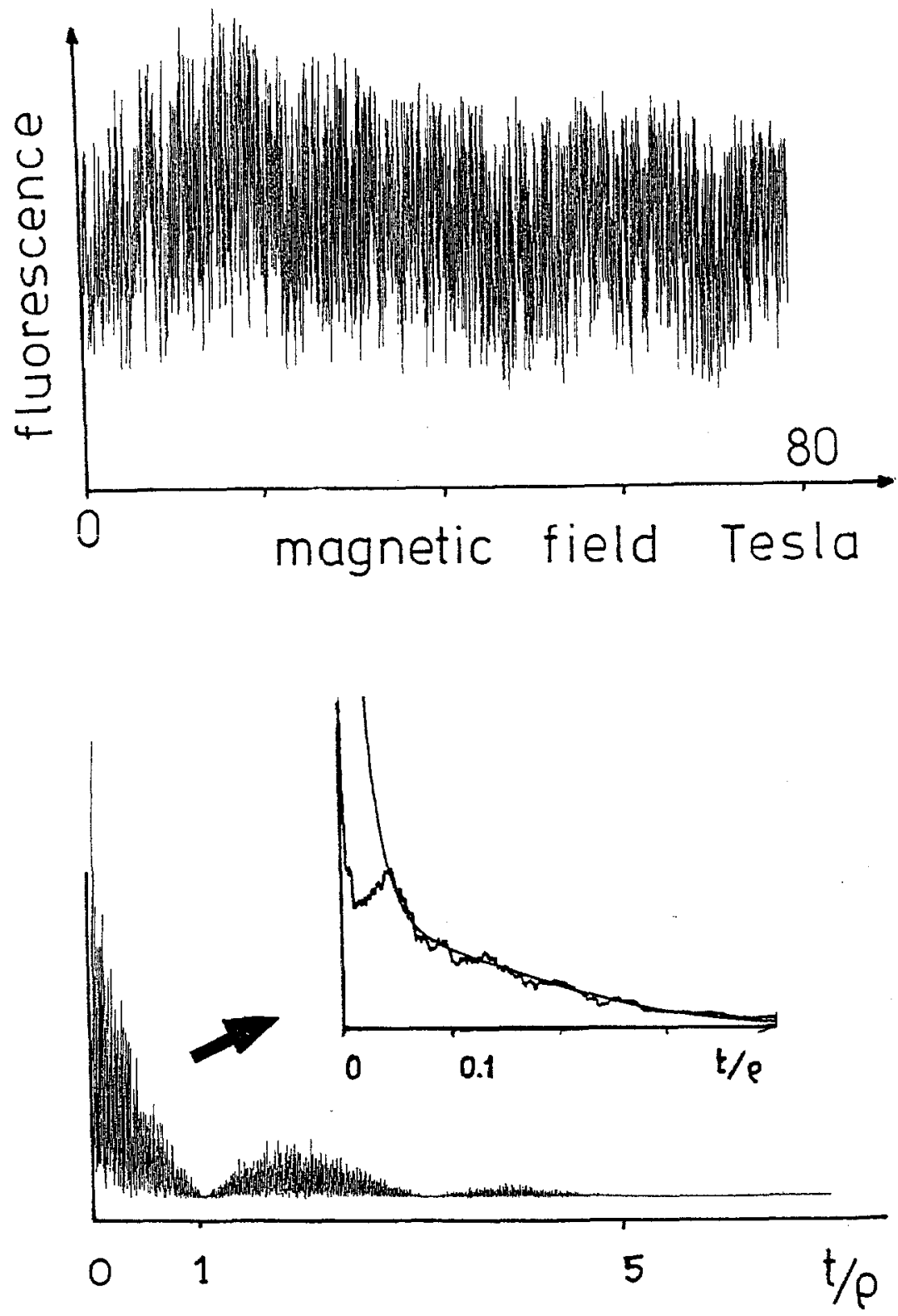

Fig. 4 : 0 to 8 Tesla anticrossing spectrum of Methylglyoxal and the unsmoothed $|F . T|$ of this spectrum. The arches are due to hyperfine doublets of triplets levels which are separated by 28 Gauss. The smoothed $|F . T|$ near the origin displays a correlation hole for $t / \rho \leqslant 0.05$. 
The F.T. of the anticrossing spectrum of glyoxal does not display any "correlation hole", we conclude that there is no significant correlation property between the vibrational levels of the $\mathrm{T}_{1}$ electronic state at $3500 \mathrm{~cm}^{-1}$. At this energy, above the vibrationless level the vibrational density of states is 1.5 levels per $\mathrm{cm}^{-1}$ per symmetry.

In contrast, significant correlation properties are observed between vibrational levels of the $T_{1}$ electronic state of methylglyoxal at $3000 \mathrm{~cm}^{-1}$ above the vibrationless level.

The 0 to 8 Tesla anticrossing spectrum of methylglyoxal $\left(\mathrm{CH}_{3}-\mathrm{CO}-\mathrm{CHO}\right)$ of figure 4 looks like noise ! However ail the features of this spectrum are reproducible. There are approximately 5000 anticrossings in this spectrum detailled below:

- The F.T. of this spectrum displays arches (fig. 4) which are due to hyperfine doublets (splitting of 28 gauss), induce by Fermi contact interaction. These arches correspond to deterministic properties of the spectrum.

- Furthermore, the $M_{S}=+1$ and $M_{S}=-1$ anticrossing spectra are superposed.

- We do not know all the anticrossing selection rules for methylglyoxal but, assuming the same kind of interaction (spin-orbitvibronic) as in glyoxal, the observed anticrossing is composed of both $N=1, K=0$ and $N=1, K=1$ components. These component may be uncoupled or coupled.

- The two vibrational symmetry classes of the $C_{s}$ point-group of methylglyoxal (or the classes of the corresponding G6 molecular symmetry group)(25) may also give two superposed anticrossing spectra.

As a result, between four and sixteen sets of independent symmetry are contained in our anticrossing spectrum.

A "correlation hole" appears in the smoothed F.T. of the anticrossing spectrum (figure 4 ) at $0 \leqslant t \leqslant 0.05$. The estimated shape of the "slow component" is also drawn. The width of the very narrow "correlation hole", 0.05 , (instead of 1 for one G.0.E. spectrum, see abovel is due to the superposition of several "pure" anticrossing spectra, as explained in chapter IV : the F.T. of a spectrum composed of m "correlated" spectra displays a "correlation hole" which is diminished in width by a factor of $\mathrm{m}$.

Furthemore, a detailled inspection of the F.T. near the origin shows that the correlations disappear for large $L(L \geqslant 100)$ or conversely for small times $t \leqslant 0,01$ (in unit of reciprocal mean spacing). 
Nevertheless, the existence of the "correlation hole" definitively shows significant correlations properties in triplet vibrational levels of methylglyoxal.

Summary and general conclusions.

The range of the number of degrees of freedom, the available range of density of states, and a variation in the coupling strength with energy make polyatomic molecules a fertile testing ground for the study of correlation properties in physical systems.

We are in the early age of the experimental study of the statistical properties of these vibrational levels. Only a few experimental molecular spectra display significant correlation properties. According to the discussion presented in chapters I to III, only two step state to state process, like optical-optical, or microwave-optical or optical-abticrossing, are able to produce spectra with the spectral purity required for the analysis of statistical correlation properties.

The understanding of these correlation properties may provide new insignts for molecular dynamics such as intramolecular vibrational redistribution and quantum chaos. The new technique of F.T. described in section IV is a crucial progress in analysis because it enables one to study large amounts of data at chemically relevant energies and complexities.

The existence of finite range of correlation properties (and the possibility of measuring very long streches of leveis in molecu1 ar physics) is a new feature not contained in G.0.E., which, by construction, have an "infinite" range of correlation properties.

\section{REFERENCES}

(1) T.A. BRODY, J. FLORES, J.B. FRENCH, P.A. MELLO, A. PANDEY and S.S. WONG.

Rev. Mod. Phys. 53385 (1981) and references cited within.

(2) M.L. MEHTA, Random Matrix (Academic, New-York 1967)

(3) G.E. POWELL and J.C. PERCIVAL

J. Phys. A : Math. Gen. $12 \mathrm{n}^{\circ} 112053$ (1979)

(4) a. O. BOHIGAS, M.J. GIANNONI and C. SCHMIT, Phys. Rev. Lett. 521 (1984)

b. E. HALLER, H. KOPPEL and L.S. CEDERBAUM Phys. Rev. Lett. 521665 (1984)

c. T.H. SELIGMAN, J.J.M. VERBAARSCHOT and M.R. ZIRNBAUER Phys. Rev. Lett. 53215 (1984) 
(5) T.H. SELIGMAN, J.J.M. VERBAARSCHOT and M.R. ZIRNBAUER

J. Phys. A : Math. Gen. 182751 (1985)

(6) J. KATO, J. Chem. Phys. 823020 (1985)

(7) R. JOST, Second Conf. on Quantum Chaos, Mexico 1986.

(8) 1. C.S. PARMENTER, J. Phys. Chem. 86 1735 (1982)

2. B. FOURMANN, C. JOUVET, A. TRAMER, J.M. LE BARS and P. MILLIE J. Chem. Phys. 9225 (1985)

(9) P. FELKER and A. ZEWAIL

J. Chem. Phys. 82 2961, 2975, 2994, 3003 (1985)

(10) L. LEVIANDIER, M. LOMBARDI, R. JOST, J.P. PIQUE Accepted in Phys. Rev. Lett. (1986)

(11) J.M. DELORY and C. TRIC, Chem. Phys. $\underline{3}, 54$ (1974)

(12) R.E. SMALLEY, L. WHARTON and D.H. LEVY J. Chem. Phys. 63, $n^{\circ} 11,4977$ (1975)

(13) E. HALLER, H. KOPPEL and L.S. CEDERBAUM J. Mo1. Spectros. 111,377 (1985)

(14) E. HALLER, H. KOPPEL and L.S. CEDERBAUM, Chem. Phys. Letters 101215 (1983)

(15) K.K. LEHMANN and S.L. COY, J. Chem. Phys. 833290 (1985)

(16) J.L. HARDWICK, J. Mo 1. SpectrosC. 10985 (1985)

(17) K.K. LEHMANN, S.L. COY, M. LOMBARDI and J.P. PIQUE to be published

(18) H.L. DAI, R.W. FIELD and J.L. KINSEY, J. Chem. Phys. 82, n4, 2161 (1985)

(19) M.I. DAVIS and E.J. HELLER J. Chem. Phys. 805036 (1984) and cited paper.

(20) E. ABRAMSON, R.W. FIELD, D. IMBRE, K.K. INNES and J.L. KINSEY J. Chem. Phys. 802298 (1984)

(21) S. MUKAMEL, J. SUE and A. PANDEY Chem. Phys. Lett. 105134 (1984)

(22) R.L. SUNDBERG, E. ABRAMSON, J.L. KINSEY and R.W. FIELD J. Chem. Phys. 83466 (1985)

(23) J. DEROUARD, R. JOST and M. LOMBARDI Journal de Physique Lettres 37, L 135 (1976)

(24) P. DUPRE, R. JOST and M. LOMBARDI Chem. Phys. 91355 (1984)

(25) P.R. BUNKER in Vibrational Spectra and Structure Vol. 3 J.R. DURIG ed. (Dekker, New-York), 1975 and in Molecular Symmetry and Spectroscopy, Acad. Press, New-York 1979 